Elect. Comm. in Probab. 6 (2001) 1-14

\title{
DONSKER-TYPE THEOREM FOR BSDES
}

\author{
PHILIPPE BRIAND \\ IRMAR, Université Rennes 1, \\ 35 042 Rennes Cedex, FRANCE \\ email: Philippe.Briand@univ-rennes1.fr \\ BERNARD DELYON \\ IRMAR, Université Rennes 1, \\ 35 042 Rennes Cedex, FRANCE \\ email: Bernard.Delyon@univ-rennes1.fr \\ JEAN MÉMIN \\ IRMAR, Université Rennes 1, \\ 35 042 Rennes Cedex, FRANCE \\ email: Jean.Memin@univ-rennes1.fr
}

submitted June 28, 2000 Final version accepted January 10, 2001

AMS 2000 Subject classification: 60H10, 60Fxx

backward stochastic differential equation (BSDE), stability of BSDEs, weak convergence of filtrations, discretization.

\section{Abstract}

This paper is devoted to the proof of Donsker's theorem for backward stochastic differential equations (BSDEs for short). The main objective is to give a simple method to discretize in time a BSDE. Our approach is based upon the notion of "convergence of filtrations" and covers the case of a $(y, z)$-dependent generator.

\section{Introduction}

We consider in this paper the following backward stochastic differential equation (BSDE for short):

$$
Y_{t}=\xi+\int_{t}^{T} f\left(Y_{s}, Z_{s}\right) d s-\int_{t}^{T} Z_{s} d W_{s}, \quad 0 \leq t \leq T,
$$

where $W$ is a standard Brownian motion. The unknowns are the adapted (w.r.t. $\mathcal{F} .^{W}$ ) processes $Y$ and $Z$. $(y, z) \longmapsto f(y, z)$ is a Lipschitz function and $\xi$ is a random variable measurable w.r.t. $\mathcal{F}_{T}^{W}$ and square integrable. It is by now well known that the BSDE (1) has a unique square integrable solution under the usual assumptions described above; see e.g. the original work of E. Pardoux and S. Peng [12] or the survey paper by N. El Karoui, M.-C. Quenez and S. PENG [7]. 
Unlike SDEs - for which a lot of approximations are available - the problem of the time discretization of BSDEs seems to be difficult: only several authors have given a contribution in this direction. Let us mention the works of V. Bally [1], D. Chevance [2, 3], J. Douglas, J. MA and Ph. Protter [6] and more recently J. MA and J. Yong [10].

In the last two works, the authors proposed numerical schemes to compute the solution of a forward-backward SDE. The method uses strongly the relationship between forward-backward SDEs and quasilinear PDEs in the spirit of the "four-step scheme" introduced by J. MA, Ph. Protter and J. Yong [9]. This requires the numerical resolution of a quasilinear PDEs.

V. Bally and D. Chevance proposed a time discretization of the BSDE (1) that avoids the resolution of a PDE. D. Chevance, in his $\mathrm{PhD}$ [3] and in his paper [2], proposed a discretization when the function $f$ does not depend on $z$. The main point is to remark that, in this case, $Y$ in the BSDE (1) is given by the equation

$$
Y_{t}=\mathbb{E}\left(\xi+\int_{t}^{T} f\left(Y_{s}\right) d s \mid \mathcal{F}_{t}^{W}\right), \quad 0 \leq t \leq T,
$$

which can be discretized in time with step-size $h=T / n$ by solving backwards in time

$$
y_{k}=\mathbb{E}\left(y_{k+1}+h f\left(y_{k+1}\right) \mid \mathcal{F}_{k}^{n}\right), \quad k=n-1, \ldots, 0
$$

and to set $Y_{t}^{n}=y_{[t / h]}$. This works if $f$ does not depend on $z$ and under reasonable assumptions, the convergence of $Y^{n}$ to $Y$ is proved $[2,3]$ together with the rate of convergence. D. Chevance gives also a space discretization to obtain a numerical scheme for solving the BSDE. Independently of the work [3], F. Coquet, V. MACkevičIus and J. MÉmin proved the convergence of the sequence $Y^{n}$ using the tool of convergence of filtrations; see [4].

The method developed by V. BALLY in [1] applies to the case where the function $f$ depends on both variables $y$ and $z$. The time discretization is performed on a random net, namely the jump times of a Poisson process. This random net appears to be one of the main arguments to overcome the difficulties due to the dependence of $f$ in the variable $z$ : it avoids to deal with the evaluations of the process $Z$ on the points of the net. In fact, the discretization concerns the term $\int_{t}^{T} f\left(Y_{s}, Z_{s}\right) d s$ while the Brownian motion and thus the stochastic integral $\int_{t}^{T} Z_{s} d W_{s}$ are not discretized.

The contribution of this paper is to prove the convergence of one of the most naive methods to discretize the BSDE (1) in the case when $f$ depends on both variables $y$ and $z$. This method consists in replacing the Brownian motion $W$ by a scaled random walk $W^{n}$ and thus the stochastic integral is also discretized. This leads to a discrete-time BSDE. This approach does not depend on the dimension (of $W$ or $Y$ ) and for simplicity, we deal with real valued processes.

To be more precise, the first step is to solve the discrete-time BSDE ( $h$ stands for $T / n$ )

$$
y_{k}=y_{k+1}+h f\left(y_{k}, z_{k}\right)-\sqrt{h} z_{k} \varepsilon_{k+1}, \quad k=n-1, \ldots, 0, \quad y_{n}=\xi^{n},
$$

where $\left\{\varepsilon_{k}\right\}_{1 \leq k \leq n}$ is an i.i.d. Bernoulli symmetric sequence, and $\xi^{n}$ is a square integrable random variable, measurable w.r.t. $\mathcal{G}_{n}$ with $\mathcal{G}_{k}=\sigma\left(\varepsilon_{1}, \ldots, \varepsilon_{k}\right)$. By a solution, we mean a discrete process $\left\{y_{k}, z_{k}\right\}_{0 \leq k \leq n-1}$, adapted w.r.t. $\mathcal{G}_{k}$. For solving (2), one chooses first

$$
z_{k}=h^{-1 / 2} \mathbb{E}\left(y_{k+1} \varepsilon_{k+1} \mid \mathcal{G}_{k}\right),
$$


and take $y_{k}$ as the solution of (2). Notice that, for $n$ large enough, $y_{k}$ is well-defined since $f$ is Lipschitz w.r.t. $y$, and that it is $\mathcal{G}_{k}$-measurable since it is $\mathcal{G}_{k+1}$-measurable and orthogonal to $\varepsilon_{k+1}$ (this would not happen if $\varepsilon$ were chosen Gaussian and it is the reason why $W^{n}$ is not chosen as the discretization of $W$ along the regular net with step-size $h$ ).

We define two continuous time processes by setting, for $0 \leq t \leq T, Y_{t}^{n}=y_{[t / h]}, Z_{t}^{n}=z_{\lfloor t / h\rfloor}$ where $\lfloor n\rfloor=(n-1)^{+}$for all integer $n$ and $\lfloor x\rfloor=[x]$ if $x$ is not an integer.

The aim of the paper is to prove the convergence of the pair $\left(Y^{n}, Z^{n}\right)$ to $(Y, Z)$; see Theorem 2.1 and its corollary below. This point of view is attractive because it consists in solving in both $y$ and $z$ a discrete BSDE. As in [4], weak convergence of filtrations will be a useful tool.

\section{Statement of the result}

Let $(\Omega, \mathcal{F}, \mathbb{P})$ be a probability space carrying a Brownian motion $\left(W_{t}\right)_{0 \leq t \leq T}$ and a sequence of i.i.d. Bernoulli sequences $\left\{\varepsilon_{k}^{n}\right\}_{1 \leq k \leq n}, n \in \mathbb{N}^{*}$. We consider, for $n \in \mathbb{N}^{*}$, the scaled random walks

$$
W_{t}^{n}=\sqrt{h} \sum_{k=1}^{[t / h]} \varepsilon_{k}^{n}, \quad 0 \leq t \leq T, \quad h=\frac{T}{n} .
$$

We will work under the following assumptions:

(H1) $f: \mathbb{R} \times \mathbb{R} \longrightarrow \mathbb{R}$ is Lipschitz i.e. for some $K \geq 0$,

$$
\forall(y, z),\left(y^{\prime}, z^{\prime}\right) \in \mathbb{R}^{2}, \quad\left|f(y, z)-f\left(y^{\prime}, z^{\prime}\right)\right| \leq K\left(\left|y-y^{\prime}\right|+\left|z-z^{\prime}\right|\right) ;
$$

$(\mathrm{H} 2) \xi$ is $\mathcal{F}_{T}^{W}$ measurable and, for all $n, \xi^{n}$ is $\mathcal{G}_{n}^{n}$ measurable where $\mathcal{G}_{k}^{n}=\sigma\left(\varepsilon_{1}^{n}, \ldots, \varepsilon_{k}^{n}\right)$ such that

$$
\mathbb{E}\left[\xi^{2}\right]+\sup _{n} \mathbb{E}\left[\left(\xi^{n}\right)^{2}\right]<\infty
$$

(H3) $\xi^{n}$ converges to $\xi$ in $\mathrm{L}^{1}$ as $n \rightarrow \infty$.

Since $f$ is Lipschitz, we can solve (for $n$ large enough) the discrete BSDE (2) where the sequence $\left\{\varepsilon_{k}\right\}_{k}$ is replaced by $\left\{\varepsilon_{k}^{n}\right\}_{k}$. If $\left\{y_{k}^{n}, z_{k}^{n}\right\}_{k}$ is the solution of this equation, we set, for $0 \leq t \leq T$, $Y_{t}^{n}=y_{[t / h]}^{n}, Z_{t}^{n}=z_{\lfloor t / h\rfloor}^{n}$. In addition, let $\left\{Y_{t}, Z_{t}\right\}_{0 \leq t \leq T}$ be the solution of the BSDE (1). We will prove the following

Theorem 2.1 Let the assumptions (H1), (H2) and (H3) hold. Let us consider the scaled random walks $W^{n}$ defined in (4). If $W^{n} \longrightarrow W$ as $n \rightarrow \infty$ in the sense that

$$
\sup _{0 \leq t \leq T}\left|W_{t}^{n}-W_{t}\right| \longrightarrow 0 \quad \text { in probability }
$$

then we have $\left(Y^{n}, Z^{n}\right) \longrightarrow(Y, Z)$ i.e.

$$
\sup _{0 \leq t \leq T}\left|Y_{t}^{n}-Y_{t}\right|^{2}+\int_{0}^{T}\left|Z_{s}^{n}-Z_{s}\right|^{2} d s \longrightarrow 0 \quad \text { as } n \rightarrow \infty \quad \text { in probability } \text {. }
$$

Remark. Theorem 2.1 can be extended to the case when $f$ depends on $t$. In such case, (2) has to be replaced by

$$
y_{k}=y_{k+1}+h f\left(k h, y_{k}, z_{k}\right)-\sqrt{h} z_{k} \varepsilon_{k+1}, \quad k=n-1, \ldots, 0,
$$


and $f$ has to be continuous in $t$.

Method for the proof. The key point is to use the following decomposition

$$
\begin{aligned}
Y^{n}-Y & =\left(Y^{n}-Y^{n, p}\right)+\left(Y^{n, p}-Y^{\infty, p}\right)+\left(Y^{\infty, p}-Y\right), \\
Z^{n}-Z & =\left(Z^{n}-Z^{n, p}\right)+\left(Z^{n, p}-Z^{\infty, p}\right)+\left(Z^{\infty, p}-Z\right),
\end{aligned}
$$

where the superscript $p$ stands for the approximation of the solution to the BSDE via the Picard method. More precisely, we set $Y^{\infty, 0}=0, Z^{\infty, 0}=0, y^{n, 0}=0, z^{n, 0}=0$ and define $\left(Y^{\infty, p+1}, Z^{\infty, p+1}\right)$ as the solution of the BSDE

$$
Y_{t}^{\infty, p+1}=\xi+\int_{t}^{T} f\left(Y_{s}^{\infty, p}, Z_{s}^{\infty, p}\right) d s-\int_{t}^{T} Z_{s}^{\infty, p+1} d W_{s}, \quad 0 \leq t \leq T,
$$

$\left(\left(Y^{\infty, p+1}, Z^{\infty, p+1}\right)\right.$ is solution of a BSDE with random coefficients $)$ and similarly

$$
y_{k}^{n, p+1}=y_{k+1}^{n, p+1}+h f\left(y_{k}^{n, p}, z_{k}^{n, p}\right)-\sqrt{h} z_{k}^{n, p+1} \varepsilon_{k+1}^{n}, \quad k=n-1, \ldots, 0, \quad y_{n}^{n, p+1}=\xi^{n} .
$$

In order to define the discrete processes on $[0, T]$ we set, for $0 \leq t \leq T, Y_{t}^{n, p}=y_{[t / h]}^{n, p}$ and $Z_{t}^{n, p}=z_{\lfloor t / h\rfloor}^{n, p}$ so that $Y^{n, p}$ is càdlàg and $Z^{n, p}$ càglàd (càdlàg means right continuous with left limits and càglàd left continuous with right limits).

We shall prove in Lemma 4.1 that the convergence of $\left(Y^{n, p}, Z^{n, p}\right)$ to $\left(Y^{n}, Z^{n}\right)$ is uniform in $n$ for the classical norm used for BSDEs which is stronger that the convergence in the sense of (5); this part is standard manipulations.

We shall prove that for any $p$, the convergence of $\left(Y^{n, p}, Z^{n, p}\right)$ to $\left(Y^{\infty, p}, Z^{\infty, p}\right)$ holds in the sense of (5); this is the difficult part of the proof, and we shall need the results of section 3 .

Remark. Let us now consider the case when $\xi^{n}=\mathbb{E}\left(\xi \mid \mathcal{G}_{n}^{n}\right)$. The convergence of $\xi^{n}$ to $\xi$ in $\mathrm{L}^{1}$ comes from Theorem 3.1. In this situation, the convergence in probability implies actually the convergence in $\mathrm{L}^{1}$ meaning the convergence of $\left(Y^{n}, Z^{n}\right)$ to $(Y, Z)$ for the norm used in the framework of BSDEs. Standard manipulations on BSDEs show that we can assume w.l.o.g that $\xi$ is in $\mathrm{L}^{\infty}$. Indeed, if it is not the case, we have, the "tilde" meaning $\xi$ and $\xi^{n}$ replaced in (1) and (2) by $\xi \mathbf{1}_{|\xi| \leq k}$ and $\mathbb{E}\left(\xi \mathbf{1}_{|\xi| \leq k} \mid \mathcal{G}_{n}^{n}\right)$, for a constant $C$ depending only on $T$ and on the Lipschitz constant $K$,

$$
\begin{gathered}
\sup _{n} \mathbb{E}\left[\sup _{0 \leq t \leq T}\left|\widetilde{Y}_{t}^{n}-Y_{t}^{n}\right|^{2}+\int_{0}^{T}\left|\widetilde{Z}_{s}^{n}-Z_{s}^{n}\right|^{2} d s\right] \leq C \mathbb{E}\left[\xi^{2} \mathbf{1}_{|\xi|>k}\right] \\
\quad \mathbb{E}\left[\sup _{0 \leq t \leq T}\left|\widetilde{Y}_{t}-Y_{t}\right|^{2}+\int_{0}^{T}\left|\widetilde{Z}_{s}-Z_{s}\right|^{2} d s\right] \leq C \mathbb{E}\left[\xi^{2} \mathbf{1}_{|\xi|>k}\right]
\end{gathered}
$$

and the last two terms can be as small as needed providing we choose $k$ large enough. But, if $\xi$ is bounded (since $f(0,0)$ is also bounded), we can prove that, for any $p \geq 2$,

$$
\sup _{n} \mathbb{E}\left[\sup _{0 \leq t \leq T}\left|Y_{t}^{n}\right|^{p}+\left(\int_{0}^{T}\left|Z_{s}^{n}\right|^{2} d s\right)^{p / 2}\right]<\infty
$$

and thus we have the convergence in $\mathrm{L}^{1}$ provided we have the convergence in probability.

In Theorem 2.1, the BSDE (1) and the discrete BSDEs were solved on the same probability space. But, we can also consider these equations on different probability spaces and obtain 
the convergence of solutions in law instead of in probability. This approach is in the spirit of Donsker's theorem.

Let us consider a standard Brownian motion $W$ defined on a probability space and a Bernoulli symmetric sequence $\left\{\varepsilon_{k}\right\}_{k \geq 1}$ defined on a possibly different probability space. We define, for each $n$, the scaled random walks

$$
S_{t}^{n}=\sqrt{h} \sum_{k=1}^{[t / h]} \varepsilon_{k}, \quad 0 \leq t \leq T, \quad \text { with } h=\frac{T}{n} .
$$

We denote by $\mathbb{D}$ the space of càdlàg (right continuous with left limits) from $[0, T]$ in $\mathbb{R}$ endowed with the topology of uniform convergence and we assume that:

(H4) $g: \mathbb{D} \longrightarrow \mathbb{R}$ is continuous and has a polynomial growth.

Let $\left\{Y_{t}, Z_{t}\right\}_{0 \leq t \leq T}$ be the solution of the BSDE (1) with $\xi=g(W)$ and let $\left\{Y_{t}^{n}, Z_{t}^{n}\right\}_{0 \leq t \leq T}$ the piecewise constant process associated with the solution of the discrete BSDE (2) with $\xi^{n}=g\left(S^{n}\right)$. We have the following corollary

Corollary 2.2 Let the assumptions (H1) and (H4) hold. Then, the sequence of processes $\left\{Y^{n}\right\}_{n}$ converges in law to $Y$ for the topology of uniform convergence on $\mathbb{D}$.

Proof. Let us notice that the laws of solution $(Y, Z)$ of $(1)$ and of $\left(y_{k}, z_{k}\right)$ of (2) depend only on $\left(\mathbb{P}_{W}, g^{-1}\left(\mathbb{P}_{W}\right), f\right)$ and $\left(\mathbb{P}_{S^{n}}, g^{-1}\left(\mathbb{P}_{S^{n}}\right), f\right)$ where $g^{-1}\left(\mathbb{P}_{W}\right)$ (resp. $\left.g^{-1}\left(\mathbb{P}_{S^{n}}\right)\right)$ is the law of $g(W)$ (resp. $g\left(S^{n}\right)$ ). So, as far as the convergence in law is concerned, we can consider the equations (1) and (2) on any probability space.

But, from Donsker's theorem and Skorokhod representation theorem, there exists a probability space, with a Brownian motion $W$ and a sequence of i.i.d. Bernoulli sequences $\left(\varepsilon^{n}\right)_{n}$ such that the processes

$$
W_{t}^{n}=\sqrt{h} \sum_{k=1}^{[t / h]} \varepsilon_{k}^{n}, \quad 0 \leq t \leq T
$$

satisfy

$$
\sup _{0 \leq t \leq T}\left|W_{t}^{n}-W_{t}\right| \longrightarrow 0, \quad \text { as } n \rightarrow \infty,
$$

in probability as well as in $\mathrm{L}^{p}$, for any $1 \leq p<\infty$.

It remains to solve the equations $(1,2)$ on this space and to apply Theorem 2.1 to obtain the convergence of $\left(Y^{n}, Z^{n}\right)$ to $(Y, Z)$ in the sense of $(5)$. This convergence implies the convergence of $\left\{Y^{n}\right\}_{n}$ to $Y$ in law for the topology of uniform convergence on $\mathbb{D}$.

\section{Convergence of filtrations}

Let us consider a sequence of càdlàg processes $W^{n}=\left(W_{t}^{n}\right)_{0 \leq t \leq T}$ and $W=\left(W_{t}\right)_{0 \leq t \leq T}$ a Brownian motion, all defined on the same probability space $(\Omega, \mathcal{G}, \mathbb{P}) ; T$ is finite. We denote by $\left(\mathcal{F}_{t}^{n}\right)$ (resp. $\left(\mathcal{F}_{t}\right)$ ) the right continuous filtration generated by $W^{n}$ (resp. $W$ ). Let us consider finally a sequence $X^{n}$ of $\mathcal{F}_{T}^{n}$-measurable integrable random variables, and $X$ an $\mathcal{F}_{T^{-}}$ measurable integrable random variable, together with the càdlàg martingales

$$
M_{t}^{n}=\mathbb{E}\left(X^{n} \mid \mathcal{F}_{t}^{n}\right), \quad M_{t}=\mathbb{E}\left(X \mid \mathcal{F}_{t}\right) .
$$

We denote by $\left[M^{n}, M^{n}\right]$ (resp. $\left.[M, M]\right)$ the quadratic variation of $M^{n}$ (resp. $M$ ) and by $\left[M^{n}, W^{n}\right]$ (resp. $\left.[M, W]\right)$ the cross variation of $M^{n}$ and $W^{n}$ (resp. $M$ and $W$ ). 
Theorem 3.1 Let us consider the following assumptions

(A1) for each $n, W^{n}$ is a square integrable $\mathcal{F}^{n}$-martingale with independent increments;

(A2) $W^{n} \longrightarrow W$ in probability for the topology of uniform convergence of càdlàg processes indexed by $t \in[0, T]$;
a. $\mathbb{E}\left[X^{2}\right]+\sup _{n} \mathbb{E}\left[\left(X^{n}\right)^{2}\right]<\infty$,
b. $\mathbb{E}\left[\left|X^{n}-X\right|\right] \longrightarrow 0$;

Then, if conditions (A1) to (A3) are satisfied, we get

$$
\left(W^{n}, M^{n},\left[M^{n}, M^{n}\right],\left[M^{n}, W^{n}\right]\right) \longrightarrow(W, M,[M, M],[M, W]) \quad \text { in probability }
$$

for the topology of uniform convergence on $[0, T]$.

Moreover, for each $t \in[0, T]$, for each $0<\delta<1$,

$$
\left(W_{t}^{n}, M_{t}^{n},\left[M^{n}, M^{n}\right]_{t}^{1 / 2},\left[M^{n}, W^{n}\right]_{t}^{1 / 2}\right) \longrightarrow\left(W_{t}, M_{t},[M, M]_{t}^{1 / 2},[M, W]_{t}^{1 / 2}\right) \quad \text { in } \mathrm{L}^{1+\delta}(\Omega, \mathcal{G}, \mathbb{P}) .
$$

Proof. For the first part, we have, from Proposition 2 in F. Coquet, J. MÉmin and L. SŁOMIŃSKI [5], the weak convergence of filtrations $\mathcal{F}^{n}$ to $\mathcal{F}$.: this means that for every $A \in \mathcal{F}_{T}$, the martingales $\mathbb{E}\left(\mathbf{1}_{A} \mid \mathcal{F}^{n}\right)$ converge in probability to $\mathbb{E}\left(\mathbf{1}_{A} \mid \mathcal{F}\right.$.) in the sense of $J_{1}$-Skorokhod topology of the space $\mathbb{D}$ of càdlàg functions. Applying now the second point of Remark 1 of [5] to $X^{n}$ and $X$, we get the convergence in probability of $M^{n}$ to $M$ in the sense of $J_{1}$-Skorokhod topology. But since $M$ is a continuous martingale this convergence is also uniform in $t$. From the assumption (A3)a, we deduce that $\sup _{n} \mathbb{E}\left[\sup _{0 \leq t \leq T}\left|M_{t}^{n}\right|^{2}\right]$ is finite and thus we have $\sup _{n} \mathbb{E}\left[\sup _{0 \leq t<T}\left|\Delta M_{t}^{n}\right|\right]<\infty$ and by assumption the same is true for the jumps of $W^{n}$. It follows, from J. JACOD [8] Theorem 1-4, that

$$
\left[M^{n}, M^{n}\right] \longrightarrow[M, M], \quad\left[M^{n}, W^{n}\right] \longrightarrow[M, W] \quad \text { in probability. }
$$

In fact, in [8], convergences are expressed as convergences in law under $J_{1}$-topology, but convergences in probability also hold, see J. MÉmin and L. SŁomiński [11] Corollary 1.9 for refinements.

Since the limit processes $W, M,[M, M]$ and $[M, W]$ are continuous, the convergence in $J_{1^{-}}$ Skorokhod topology for each component gives the uniform convergence in $t$ of the quadruplet. The second point of the theorem comes from the boundedness in $\mathrm{L}^{2}(\Omega, \mathcal{G}, \mathbb{P})$ of the sequence $\left(W_{t}^{n}, M_{t}^{n},\left[M^{n}, M^{n}\right]_{t}^{1 / 2},\left[M^{n}, W^{n}\right]_{t}^{1 / 2}\right)$ for $t$ fixed.

Using Theorem 3.1, we get the following result which is one of the key point in the proof of our main result.

Corollary 3.2 Let $W$ and $W^{n}, n \in \mathbb{N}^{*}$, be the standard Brownian motion and the random walks of Theorem 2.1. Let us consider, on the same space, $X$ and $X^{n}$ satisfying the assumption (A3) of Theorem 3.1.

Then there exists a sequence $\left(Z_{t}^{n}\right)_{0 \leq t \leq T}$ of $\mathcal{F}^{n}$-predictable processes, and an $\mathcal{F}$.-predictable process $\left(Z_{t}\right)_{0 \leq t \leq T}$ such that:

$$
\forall t \in[0, T], \quad M_{t}^{n}=\mathbb{E}\left[X^{n}\right]+\int_{0}^{t} Z_{s}^{n} d W_{s}^{n}, \quad M_{t}=\mathbb{E}[X]+\int_{0}^{t} Z_{s} d W_{s},
$$


and

$$
\int_{0}^{T}\left(Z_{t}^{n}-Z_{t}\right)^{2} d t \longrightarrow 0 \quad \text { in probability }
$$

Moreover, if $0<\delta<1, Z^{n}$ converges to $Z$ in the space $\mathrm{L}^{1+\delta}(\Omega \times[0, T], \mathcal{G} \times \mathcal{B}([0, T]), \mathbb{P} \otimes \lambda)$ where $\lambda$ denotes the Lebesgue measure on $([0, T], \mathcal{B}([0, T]))$.

Proof. The first part is completely classic: the predictable representation of $\mathcal{F}^{n}$-martingales in terms of stochastic integrals w.r.t. $W^{n}$, and of $\mathcal{F}$.-martingales in terms of stochastic integrals w.r.t. the Brownian motion $W$.

Setting $A_{t}^{n}:=h[t / h]$ and applying the first part of the previous theorem, we obtain

$$
\sup _{0 \leq t \leq T}\left|\int_{0}^{t} Z_{s}^{n} d A_{s}^{n}-\int_{0}^{t} Z_{s} d s\right| \stackrel{\mathrm{P}}{\longrightarrow} 0, \quad \sup _{0 \leq t \leq T}\left|\int_{0}^{t}\left(Z_{s}^{n}\right)^{2} d A_{s}^{n}-\int_{0}^{t} Z_{s}^{2} d s\right| \stackrel{\mathrm{P}}{\longrightarrow} 0 .
$$

From these uniform (in $t$ ) convergences, we deduce that

$$
\sup _{0 \leq t \leq T}\left|\int_{0}^{t} Z_{s}^{n} d s-\int_{0}^{t} Z_{s} d s\right| \stackrel{\mathrm{P}}{\longrightarrow} 0, \quad \sup _{0 \leq t \leq T}\left|\int_{0}^{t}\left(Z_{s}^{n}\right)^{2} d s-\int_{0}^{t} Z_{s}^{2} d s\right| \stackrel{\mathrm{P}}{\longrightarrow} 0 .
$$

Extracting a subsequence (still indexed by $n$ ), we have for almost every $\omega$,

$$
\sup _{0 \leq t \leq T}\left|\int_{0}^{t} Z_{s}^{n}(\omega) d s-\int_{0}^{t} Z_{s}(\omega) d s\right| \longrightarrow 0, \quad \sup _{0 \leq t \leq T}\left|\int_{0}^{t}\left(Z_{s}^{n}\right)^{2}(\omega) d s-\int_{0}^{t} Z_{s}^{2}(\omega) d s\right| \longrightarrow 0
$$

which implies the convergence of $Z^{n}(\omega)$ to $Z .(\omega)$ weakly in $\mathrm{L}^{2}([0, T], \lambda)$. Since $\left\|Z^{n}(\omega)\right\|_{\mathrm{L}^{2}([0, T], \lambda)}$ tends to $\|Z .(\omega)\|_{\mathrm{L}^{2}([0, T], \lambda)}$ the convergence hold actually strongly in $\mathrm{L}^{2}([0, T], \lambda)$. This gives the first part of the result.

The last result comes immediately with the $\mathrm{L}^{2}$-boundedness of $\left\{\left(\int_{0}^{T}\left(Z_{s}^{n}\right)^{2} d s\right)^{1 / 2}\right\}_{n}$.

\section{Proof of Theorem 2.1}

Equations $(6,7)$ with the following lemma proved in appendix

Lemma 4.1 With the notations following $(8,9)$,

$$
\sup _{n} \mathbb{E}\left[\sup _{0 \leq t \leq T}\left|Y_{t}^{n}-Y_{t}^{n, p}\right|^{2}+\int_{0}^{T}\left|Z_{t}^{n}-Z_{t}^{n, p}\right|^{2} d t\right] \longrightarrow 0, \quad \text { as } \quad p \rightarrow \infty .
$$

imply that it remains to prove the convergence to zero of the process $Y^{n, p}-Y^{\infty, p}$ and $Z^{n, p}-$ $Z^{\infty, p}$. This will be done by induction on $p$. For sake of clarity, we drop the superscript $p$, set the time in subscript and write everything in continuous time, so that equations $(8,9)$ become

$$
\begin{aligned}
Y_{t}^{\prime} & =\xi+\int_{t}^{T} f\left(Y_{s}, Z_{s}\right) d s-\int_{t}^{T} Z_{s}^{\prime} d W_{s}, \quad 0 \leq t \leq T, \\
Y_{t}^{\prime n} & =\xi^{n}+\int_{t}^{T} f\left(Y_{s-}^{n}, Z_{s}^{n}\right) d A_{s}^{n}-\int_{t}^{T} Z_{s}^{\prime n} d W_{s}^{n}, \quad 0 \leq t \leq T,
\end{aligned}
$$


where $A_{s}^{n}=[s / h] h$ and $Y_{-}$denotes the càglàd process associated to $Y$. The assumption is that $\left\{Y_{t}^{n}, Z_{t}^{n}\right\}_{0 \leq t \leq T}$ converges to $\left\{Y_{t}, Z_{t}\right\}_{0 \leq t \leq T}$ in the sense of (5) and we have to prove that $\left\{Y_{t}^{\prime n}, Z_{t}^{\prime n}\right\}_{0 \leq t \leq T}$ converges to $\left\{Y_{t}^{\prime}, Z_{t}^{\prime}\right\}_{0 \leq t \leq T}$ in the same sense.

The process, defined by

$$
M_{t}^{n}=Y_{t}^{\prime n}+\int_{0}^{t} f\left(Y_{s-}^{n}, Z_{s}^{n}\right) d A_{s}^{n}, \quad 0 \leq t \leq T
$$

satisfies

$$
M_{t}^{n}=M_{0}^{n}+\int_{0}^{t} Z_{s}^{\prime n} d W_{s}^{n}
$$

Hence $M^{n}$ is an $\mathcal{F}^{n}$-martingale and, since $Y_{T}^{n}=\xi^{n}$,

$$
M_{t}^{n}=\mathbb{E}\left(M_{T}^{n} \mid \mathcal{F}_{t}^{n}\right), \quad M_{T}^{n}=Y_{T}^{n}+\int_{0}^{T} f\left(Y_{s-}^{n}, Z_{s}^{n}\right) d A_{s}^{n} .
$$

If we want to apply Corollary 3.2 , we have to prove the $\mathrm{L}^{1}$ convergence of $M_{T}^{n}$. But since $Y^{n}$ and $Z^{n}$ are piecewise constant, we have

$$
\begin{aligned}
\left|M_{T}^{n}-Y_{T}-\int_{0}^{T} f\left(Y_{s}, Z_{s}\right) d s\right| & \leq\left|Y_{T}^{n}-Y_{T}\right|+\int_{0}^{T}\left|f\left(Y_{s}^{n}, Z_{s}^{n}\right)-f\left(Y_{s}, Z_{s}\right)\right| d s \\
& \leq(1+K T) \sup _{0 \leq t \leq T}\left|Y_{t}^{n}-Y_{t}\right|+K \int_{0}^{T}\left|Z_{s}^{n}-Z_{s}\right| d s,
\end{aligned}
$$

which tends to zero in probability and then in $\mathrm{L}^{1}$ by $\mathrm{L}^{2}$-boundedness. This and equations $(12,13)$, imply together with Corollary 3.2 that $M^{n}$ converges to

$$
M_{t}=\mathbb{E}\left(Y_{T}+\int_{0}^{T} f\left(Y_{s}, Z_{s}\right) d s \mid \mathcal{F}_{t}\right)=Y_{t}^{\prime}+\int_{0}^{t} f\left(Y_{s}, Z_{s}\right) d s
$$

in the sense that

$$
\sup _{0 \leq t \leq T}\left|M_{t}^{n}-M_{t}\right|+\int_{0}^{T}\left|Z_{s}^{\prime n}-Z_{s}^{\prime}\right|^{2} d s \longrightarrow 0 \quad \text { in probability. }
$$

Since we want to prove that

$$
\sup _{0 \leq t \leq T}\left|Y_{t}^{\prime n}-Y_{t}^{\prime}\right|+\int_{0}^{T}\left|Z_{s}^{\prime n}-Z_{s}^{\prime}\right|^{2} d s \longrightarrow 0 \quad \text { in probability }
$$

it remain only to demonstrate

$$
\sup _{0 \leq t \leq T}\left|\int_{0}^{t} f\left(Y_{s}^{n}, Z_{s}^{n}\right) d A_{s}^{n}-\int_{0}^{t} f\left(Y_{s}, Z_{s}\right) d s\right| \longrightarrow 0 \quad \text { in probability. }
$$

This is true since we have just proved the convergence of $\int_{0}^{T}\left|f\left(Y_{s}^{n}, Z_{s}^{n}\right)-f\left(Y_{s}, Z_{s}\right)\right| d s$ to zero in probability and since the jumps of $t \mapsto \int_{0}^{t} f\left(Y_{s}^{n}, Z_{s}^{n}\right) d A_{s}^{n}$ tends to zero according to (10). 


\section{Discrete BSDEs and PDEs}

In this section, we give an application of Theorem 2.1 to BSDEs in a Markovian framework which are related to semilinear PDEs. Let us first recall the relations between BSDEs and PDEs. The setup is the following: let $b$ and $\sigma$ be two functions defined on $[0, T] \times \mathbb{R}$ with values in $\mathbb{R} ; f$ is a function defined on $[0, T] \times \mathbb{R}^{3}$ in $\mathbb{R}$ and $g$ from $\mathbb{R}$ to $\mathbb{R}$. We assume that these functions are $K$-Lipschitz continuous w.r.t. all their variables.

Let us introduce the unique (in the class of continuous functions with polynomial growth) viscosity solution to the $\mathrm{PDE}$ on $[0, T] \times \mathbb{R}$,

$$
\partial_{t} U+\frac{1}{2} \sigma^{2}(t, x) \partial_{x, x} U+b(t, x) \partial_{x} U+f\left(t, x, U, \sigma(t, x) \partial_{x} U\right)=0, \quad U(T, \cdot)=g(\cdot) .
$$

It is a very well known fact - we refer to S. PENG [14] for classical solutions and to E. PARdoux, S. PEng [13] for viscosity solutions - that $U$ is related to the following BSDE: for $x \in \mathbb{R},\left\{\left(Y_{t}, Z_{t}\right)\right\}_{0 \leq t \leq T}$ is the solution to the equation

$$
Y_{t}=g\left(X_{T}\right)+\int_{t}^{T} f\left(r, X_{r}, Y_{r}, Z_{r}\right) d r-\int_{r}^{T} Z_{r} d W_{r}, \quad 0 \leq t \leq T
$$

the terminal condition in this BSDE is of the special form $g\left(X_{T}\right)$ where $\left\{X_{t}\right\}_{0 \leq t \leq T}$ is the solution to the SDE

$$
X_{t}=x+\int_{0}^{t} b\left(r, X_{r}\right) d r+\int_{0}^{t} \sigma\left(r, X_{r}\right) d W_{r}, \quad 0 \leq t \leq T .
$$

By the nonlinear Feynman-Kac formula, we have:

$$
\forall t \in[0, T], \quad U\left(t, X_{t}\right)=Y_{t}
$$

In the remaining of this section, we will use the result of Theorem 2.1 to discretize the solution to the BSDE (15) and then to construct an approximation of the solution $U$ to the PDE (14) which solves a discrete PDE.

The framework is the same as in the section $2:(\Omega, \mathcal{F}, \mathbb{P})$ is a probability space carrying a Brownian motion $\left(W_{t}\right)_{0 \leq t \leq T}$ and a sequence of i.i.d. Bernoulli sequences $\left\{\varepsilon_{k}^{n}\right\}_{1 \leq k \leq n}, n \in \mathbb{N}^{*}$. We consider, for $n \in \mathbb{N}^{*}$, the scaled random walks

$$
W_{t}^{n}=\sqrt{h} \sum_{k=1}^{[t / h]} \varepsilon_{k}^{n}, \quad 0 \leq t \leq T
$$

and we assume that

$$
\sup _{0 \leq t \leq T}\left|W_{t}^{n}-W_{t}\right| \longrightarrow 0 \quad \text { in probability, }
$$

as well as in $\mathrm{L}^{p}$ for all real $p \geq 1$. This is not a restriction as explained in Corollary 2.2. We define also $\mathcal{G}_{k}^{n}=\sigma\left(\varepsilon_{1}^{n}, \ldots, \varepsilon_{k}^{n}\right)$.

We consider the time discretization of the interval $[0, T]$ with step-size $T / n$; we pick $n$ such that $K T / n<1$ and we set $h=T / n$ so that $K h<1$.

We fix a real $x .\left\{\chi_{i}^{n}\right\}_{i}$ is defined by the relation

$$
\chi_{0}^{n}=x, \quad \chi_{i+1}^{n}=\chi_{i}^{n}+h b\left((i+1) h, \chi_{i}^{n}\right)+\sqrt{h} \sigma\left((i+1) h, \chi_{i}^{n}\right) \varepsilon_{i+1}^{n}, \quad i=k, \ldots, n-1 .
$$


$\left\{\chi_{i}^{n}\right\}_{0 \leq i \leq n}$ is $\left\{\mathcal{G}_{i}^{n}\right\}_{i}$-measurable. To define this process in continuous time, we set,

$$
\forall t \in[0, T], \quad X_{t}^{n}=\chi_{[t / h]}^{n} .
$$

It is worth noting that the process $\left\{X_{t}^{n}\right\}_{0 \leq t \leq T}$ is the strong solution to the SDE

$$
X_{t}^{n}=x+\int_{0}^{t} b\left(r, X_{r-}^{n}\right) d\left[W^{n}, W^{n}\right]_{r}+\int_{0}^{t} \sigma\left(r, X_{r-}^{n}\right) d W_{r}^{n}, \quad 0 \leq t \leq T ;
$$

Hence, we are in the classical context of convergence of solutions to SDEs. We refer to L. SŁOMIŃsKi [15] for general results in this area.

We solve the discrete BSDE $-\left\{y_{i}^{n}, z_{i}^{n}\right\}_{i}$ is the $\left\{\mathcal{G}_{i}^{n}\right\}_{i}$-adapted solution -

$$
y_{i}^{n}=y_{i+1}^{n}+h f\left((i+1) h, \chi_{i}^{n}, y_{i}^{n}, z_{i}^{n}\right)-\sqrt{h} z_{i}^{n} \varepsilon_{i+1}^{n}, \quad i=0, \ldots, n-1, \quad y_{n}^{n}=g\left(\chi_{n}^{n}\right) .
$$

Similarly to the previous SDE, if we set

$$
\forall t \in[0, T], \quad Y_{t}^{n}=y_{[t / h]}^{n}, \quad Z_{t}^{n}=Z_{\lfloor t / h\rfloor}^{n},
$$

the equation (18) can be rewritten as

$$
Y_{t}^{n}=g\left(X_{T}^{n}\right)+\int_{t}^{T} f\left(r, X_{r-}^{n}, Y_{r-}^{n}, Z_{r}^{n}\right) d\left[W^{n}, W^{n}\right]_{r}-\int_{t}^{T} Z_{r}^{n} d W_{r}^{n}, \quad 0 \leq t \leq T .
$$

Let $\mathrm{D}_{-}^{n}$ and $\mathrm{D}_{+}^{n}$ be the following discrete operators:

$$
\begin{aligned}
& \mathrm{D}_{+}^{n} u(k, x)=\frac{1}{2}\{u(k, x+h b(k h, x)+\sqrt{h} \sigma(k h, x))+u(k, x+h b(k h, x)-\sqrt{h} \sigma(k h, x))\}, \\
& \mathrm{D}_{-}^{n} u(k, x)=\frac{1}{2}\{u(k, x+h b(k h, x)+\sqrt{h} \sigma(k h, x))-u(k, x+h b(k h, x)-\sqrt{h} \sigma(k h, x))\} .
\end{aligned}
$$

We have the following result:

Proposition 5.1 Let $u^{n}$ solves the following discrete (in time) PDE: for each $x$,

$u^{n}(k, x)-h f\left((k+1) h, x, u^{n}(k, x), h^{-1 / 2} \mathrm{D}_{-}^{n} u^{n}(k+1, x)\right)=\mathrm{D}_{+}^{n} u^{n}(k+1, x), \quad k=0, \ldots, n-1$, with the terminal condition $u^{n}(n, x)=g(x)$. Then, we have,

$$
\forall k=0, \ldots, n-1, \quad y_{k}^{n}=u^{n}\left(k, \chi_{k}^{n}\right), \quad z_{k}^{n}=h^{-1 / 2} \mathrm{D}_{-}^{n} u^{n}\left(k+1, \chi_{k}^{n}\right) .
$$

Proof. Suppose that $y_{k+1}^{n}=u^{n}\left(k+1, \chi_{k+1}^{n}\right)$ for some $k \in\{0, \ldots, n-1\}$. From the equation (18), we have

$$
z_{k}^{n}=h^{-1 / 2} \mathbb{E}\left[u^{n}\left(k+1, \chi_{k+1}^{n}\right) \varepsilon_{k+1}^{n} \mid \mathcal{G}_{k}^{n}\right]=h^{-1 / 2} \mathrm{D}_{-}^{n} u^{n}\left(k+1, \chi_{k}^{n}\right),
$$

and then, since $\mathbb{E}\left[u^{n}\left(k+1, \chi_{k+1}^{n}\right) \mid \mathcal{G}_{k}^{n}\right]=\mathrm{D}_{+}^{n} u^{n}\left(k+1, \chi_{k}^{n}\right)$,

$$
y_{k}^{n}=\mathrm{D}_{+}^{n} u^{n}\left(k+1, \chi_{k}^{n}\right)+h f\left((k+1) h, \chi_{k}^{n}, y_{k}^{n}, h^{-1 / 2} \mathrm{D}_{-}^{n} u^{n}\left(k+1, \chi_{k}^{n}\right)\right) .
$$

Noting that $f$ is $K$-Lipschitz and that $K h<1$, we get $y_{k}^{n}=u^{n}\left(k, \chi_{k}^{n}\right)$.

The proof is thus complete by induction since obviously $u^{n}\left(n, \chi_{n}^{n}\right)=g\left(\chi_{n}^{n}\right)=y_{n}^{n}$.

We define a new sequence of functions by setting

$$
\forall t \in[0, T], \forall x \in \mathbb{R}, \quad U^{n}(t, x)=u^{n}([t / h], x),
$$

and we are interested in the convergence of the sequence $\left\{U^{n}\right\}_{n}$. 
Theorem 5.2 For each $x \in \mathbb{R}, U^{n}(0, x)$ converges to $U(0, x)$, $U$ being the solution to the semilinear PDE (14). This convergence is uniform on compact sets.

Proof. We fix $x \in \mathbb{R}$. We have $U^{n}(0, x)=Y_{0}^{n}$ and also $U(0, x)=Y_{0}$. As a consequence the proof of the first statement will be finished if we are able to prove that

$$
\mathbb{E}\left[\sup _{0 \leq r \leq T}\left|Y_{r}^{n}-Y_{r}\right|^{2}\right] \longrightarrow 0, \quad \text { as } n \longrightarrow \infty .
$$

This follows from slight adaptations of Theorem 2.1 since, from L. SŁomiński [15, Theorem 3.1 , we know that

$$
\mathbb{E}\left[\sup _{0 \leq r \leq T}\left|X_{r}^{n}-X_{r}\right|^{2}\right] \longrightarrow 0, \quad \text { as } n \longrightarrow \infty .
$$

For the proof of the uniform convergence on compact sets, we first remark that since $f$ is Lipschitz, for each compact $\mathcal{K}$, there exists a constant $C$ such that, for all $x, x^{\prime}$ in $\mathcal{K}$,

$$
\sup _{n} \mathbb{E}\left[\sup _{0 \leq r \leq T}\left|Y_{r}^{n}(x)-Y_{r}^{n}\left(x^{\prime}\right)\right|^{2}\right] \leq C\left|x-x^{\prime}\right|^{2},
$$

and the same is true for $Y(x)$ in place of $Y^{n}(x)$. The last inequality is derived from the similar inequality for $X^{n}(x)$ (see [15]).

Let us fix a compact $\mathcal{K}$. For each $k \in \mathbb{N}^{*}$, we can find a finite set of points of $\mathcal{K}$ say $\mathcal{K}_{k}$ such that for each point $x \in \mathcal{K}$ there exists a point $x_{k} \in \mathcal{K}_{k}$ such that $\left|x-x_{k}\right| \leq 1 / k$. Let $x \in \mathcal{K}$; We have from (19),

$$
\mathbb{E}\left[\sup _{0 \leq r \leq T}\left|Y_{r}^{n}(x)-Y_{r}(x)\right|^{2}\right] \leq 3 \sup _{x \in \mathcal{K}_{k}} \mathbb{E}\left[\sup _{0 \leq r \leq T}\left|Y_{r}^{n}(x)-Y_{r}^{n}(x)\right|^{2}\right]+6 C / k^{2},
$$

and thus, $\mathcal{K}_{k}$ being finite,

$$
\limsup _{n \rightarrow \infty} \sup _{x \in \mathcal{K}} \mathbb{E}\left[\sup _{0 \leq r \leq T}\left|Y_{r}^{n}(x)-Y_{r}(x)\right|^{2}\right] \leq 6 C / k^{2}
$$

which gives the result since $k$ is arbitrary.

Remark. As in the continuous time case, we can construct the function $u^{n}$ from the discrete SDE and BSDE $(17,18)$ if we let the diffusion start at time $s$ instead of time 0 . An easy consequence is that the sequence of functions $U^{n}$ converges to $U$ uniformly on compact sets of $[0, T] \times \mathbb{R}$ and not only at time $t=0$ as proved just before. We choose to present the result only for $t=0$ to avoid a lot of notations coming from the flow generated by a SDE.

\section{A Proof of Lemma 4.1}

For the proof of this lemma we come back to the discrete notations and we show that

Lemma A.1 There exist $\alpha>1$ and $n_{0} \in \mathbb{N}$ such that for all $n \geq n_{0}$, for all $p \in \mathbb{N}^{*}$,

$$
\left\|\left(y^{n, p+1}-y^{n, p}, z^{n, p+1}-z^{n, p}\right)\right\|_{\alpha}^{2} \leq \frac{2}{3}\left\|\left(y^{n, p}-y^{n, p-1}, z^{n, p}-z^{n, p-1}\right)\right\|_{\alpha}^{2},
$$


where, for $p \in \mathbb{N}$,

$\left\|\left(y^{n, p+1}-y^{n, p}, z^{n, p+1}-z^{n, p}\right)\right\|_{\alpha}^{2}:=\mathbb{E}\left[\sup _{0 \leq k \leq n} \alpha^{k h}\left|y_{k}^{n, p+1}-y_{k}^{n, p}\right|^{2}+h \sum_{k=0}^{n-1} \alpha^{k h}\left|z_{k}^{n, p+1}-z_{k}^{n, p}\right|^{2}\right]$.

Proof. For notational convenience, let us write $y, z$ in place of $y^{n, p+1}-y^{n, p}, z^{n, p+1}-z^{n, p}$ and $u, v$ in place of $y^{n, p}-y^{n, p-1}, z^{n, p}-z^{n, p-1}$. Let us pick $\beta>1$ to be chosen later. With these notations in hands, we have, for $k=0, \ldots, n-1$, since $y_{n}=0$,

$$
\beta^{k} y_{k}^{2}=\sum_{i=k}^{n-1} \beta^{i} y_{i}^{2}-\beta^{i+1} y_{i+1}^{2}=(1-\beta) \sum_{i=k}^{n-1} \beta^{i} y_{i}^{2}+\beta \sum_{i=k}^{n-1} \beta^{i}\left(y_{i}^{2}-y_{i+1}^{2}\right) .
$$

We write $y_{i}^{2}-y_{i+1}^{2}=2 y_{i}\left(y_{i}-y_{i+1}\right)-\left(y_{i}-y_{i+1}\right)^{2}$, to use the equation (9), since

$$
y_{i}-y_{i+1}=h\left\{f\left(y_{i}^{n, p}, z_{i}^{n, p}\right)-f\left(y_{i}^{n, p-1}, z_{i}^{n, p-1}\right)\right\}-\sqrt{h} z_{i} \varepsilon_{i+1}^{n} .
$$

Since $f$ is Lipschitz in $(y, z)$ with constant $K$, we have, for each $\nu>0$,

$$
2 y_{i}\left\{f\left(y_{i}^{n, p}, z_{i}^{n, p}\right)-f\left(y_{i}^{n, p-1}, z_{i}^{n, p-1}\right)\right\} \leq 2 K\left|y_{i}\right|\left(\left|u_{i}\right|+\left|v_{i}\right|\right) \leq 2\left(K^{2} / \nu\right) y_{i}^{2}+\nu\left(u_{i}^{2}+v_{i}^{2}\right),
$$

and moreover, (20) implies easily that

$$
h z_{i}^{2} \leq 2\left(y_{i}-y_{i+1}\right)^{2}+4 K^{2} h^{2}\left(u_{i}^{2}+v_{i}^{2}\right) .
$$

As a byproduct of these inequalities, we deduce that, for $k=0, \ldots, n-1$,

$$
\begin{aligned}
2 \sum_{i=k}^{n-1} \beta^{i} y_{i}\left(y_{i}-y_{i+1}\right) & \leq 2 K^{2}(h / \nu) \sum_{i=k}^{n-1} \beta^{i} y_{i}^{2}+\nu h \sum_{i=k}^{n-1} \beta^{i}\left(u_{i}^{2}+v_{i}^{2}\right)-2 \sqrt{h} \sum_{i=k}^{n-1} \beta^{i} y_{i} z_{i} \varepsilon_{i+1}^{n}, \\
-\sum_{i=k}^{n-1} \beta^{i}\left(y_{i}-y_{i+1}\right)^{2} & \leq-(h / 2) \sum_{i=k}^{n-1} \beta^{i} z_{i}^{2}+2 K^{2} h^{2} \sum_{i=k}^{n-1} \beta^{i}\left(u_{i}^{2}+v_{i}^{2}\right),
\end{aligned}
$$

and, setting $\rho=\left(\nu+2 K^{2} h\right) \beta h$, we get

$\beta^{k} y_{k}^{2}+\beta(h / 2) \sum_{i=k}^{n-1} \beta^{i} z_{i}^{2} \leq\left(1-\beta+2 K^{2} h \beta / \nu\right) \sum_{i=k}^{n-1} \beta^{i} y_{i}^{2}-2 \beta \sqrt{h} \sum_{i=k}^{n-1} \beta^{i} y_{i} z_{i} \varepsilon_{i+1}^{n}+\rho \sum_{i=k}^{n-1} \beta^{i}\left(u_{i}^{2}+v_{i}^{2}\right)$.

Thus, if $1-\beta+2 K^{2} h \beta / \nu \leq 0$, we have, for $k=0, \ldots, n-1$,

$$
\beta^{k} y_{k}^{2}+\beta(h / 2) \sum_{i=k}^{n-1} \beta^{i} z_{i}^{2} \leq \rho \sum_{i=0}^{n-1} \beta^{i}\left(u_{i}^{2}+v_{i}^{2}\right)-2 \beta \sqrt{h} \sum_{i=k}^{n-1} \beta^{i} y_{i} z_{i} \varepsilon_{i+1}^{n}
$$

in particular, taking the expectation of the previous inequality for $k=0$, we get

$$
\mathbb{E}\left[\sum_{i=0}^{n-1} \beta^{i} z_{i}^{2}\right] \leq 2\left(\nu+2 K^{2} h\right) \mathbb{E}\left[\sum_{i=0}^{n-1} \beta^{i}\left(u_{i}^{2}+v_{i}^{2}\right)\right] .
$$


Now, coming back to (21), we have, since $y_{n}=0$,

$$
\sup _{0 \leq k \leq n} \beta^{k} y_{k}^{2} \leq \rho \sum_{i=0}^{n-1} \beta^{i}\left(u_{i}^{2}+v_{i}^{2}\right)+4 \beta \sqrt{h} \sup _{0 \leq k \leq n-1}\left|\sum_{i=0}^{k} \beta^{i} y_{i} z_{i} \varepsilon_{i+1}^{n}\right|,
$$

and using Burkholder-Davis-Gundy inequality, we obtain, for a universal constant $C$,

$$
\begin{aligned}
\mathbb{E}\left[\sup _{0 \leq k \leq n} \beta^{k} y_{k}^{2}\right] & \leq \rho \mathbb{E}\left[\sum_{i=0}^{n-1} \beta^{i}\left(u_{i}^{2}+v_{i}^{2}\right)\right]+C \sqrt{h} \beta \mathbb{E}\left[\left(\sum_{i=0}^{n-1} \beta^{2 i} y_{i}^{2} z_{i}^{2}\right)^{1 / 2}\right] \\
& \leq \rho \mathbb{E}\left[\sum_{i=0}^{n-1} \beta^{i}\left(u_{i}^{2}+v_{i}^{2}\right)\right]+C^{2} \beta^{2}(h / 2) \mathbb{E}\left[\sum_{i=0}^{n-1} \beta^{i} z_{i}^{2}\right]+\frac{1}{2} \mathbb{E}\left[\sup _{0 \leq k \leq n} \beta^{k} y_{k}^{2}\right] .
\end{aligned}
$$

Finally, from (22), we get the inequality,

$$
\mathbb{E}\left[\sup _{0 \leq k \leq n} \beta^{k} y_{k}^{2}+h \sum_{i=0}^{n-1} \beta^{i} z_{i}^{2}\right] \leq \lambda \mathbb{E}\left[\sup _{0 \leq k \leq n} \beta^{k} u_{k}^{2}+h \sum_{i=0}^{n-1} \beta^{i} v_{i}^{2}\right],
$$

where $\lambda=2\left(\nu+2 K^{2} h\right)\left(1+\beta+C^{2} \beta^{2}\right)(1 \vee T)$, and providing that $1-\beta+2 K^{2} h \beta / \nu \leq 0$.

Firstly, we choose $\nu$ such that $2 \nu\left(2+C^{2}\right)(1 \vee T)=1 / 2$. We consider only $n$ greater than $n_{1}$ (i.e. $K h<1$ and $2 K^{2} h / \nu<1$ ). Let us pick $\beta$ of the form $\alpha^{h}$ with $\alpha \geq 1$. We want that $1-\alpha^{h}+2 K^{2} h \alpha^{h} / \nu \leq 0$ meaning that $\alpha \geq \exp \left\{-h^{-1} \log \left(1-2 K^{2} h / \nu\right)\right\}$. Since $\exp \left\{-h^{-1} \log (1-\right.$ $\left.\left.2 K^{2} h / \nu\right)\right\}$ tends to $\exp \left\{2 K^{2} / \nu\right\}$ as $n \rightarrow \infty(h \rightarrow 0)$, we choose $\alpha=\exp \left\{1+2 K^{2} / \nu\right\}$. Hence, for $n$ greater than $n_{2}$ the condition is satisfied and (23) holds for $\beta=\alpha^{h}$. It remains to observe that, $\nu$ and $\alpha$ being fixed as explained above, $\lambda$ converges, as $n \rightarrow \infty$, to $2 \nu\left(2+C^{2}\right)(1 \vee T)$ which is equal to $1 / 2$. It follows that for $n$ large enough, say $n \geq n_{0}, \lambda \leq 2 / 3$ and

$$
\mathbb{E}\left[\sup _{0 \leq k \leq n} \alpha^{k h} y_{k}^{2}+h \sum_{i=0}^{n-1} \alpha^{i h} z_{i}^{2}\right] \leq \frac{2}{3} \mathbb{E}\left[\sup _{0 \leq k \leq n} \alpha^{k h} u_{k}^{2}+h \sum_{i=0}^{n-1} \alpha^{i h} v_{i}^{2}\right],
$$

which concludes the proof of this technical lemma.

To complete the proof of Lemma 4.1, it remains to check that

$$
\sup _{n} \mathbb{E}\left[\sup _{0 \leq k \leq n-1}\left|y_{k}^{n, 1}\right|^{2}+h \sum_{i=0}^{n-1}\left|z_{i}^{n, 1}\right|^{2}\right]
$$

is finite. But it is plain to check (using the same computations as above) that for $n$ large enough,

$$
\mathbb{E}\left[\sup _{0 \leq k \leq n-1}\left|y_{k}^{n, 1}\right|^{2}+h \sum_{i=0}^{n-1}\left|z_{i}^{n, 1}\right|^{2}\right] \leq C \exp (2 T)\left(\mathbb{E}\left[\xi^{2}\right]+3 T|f(0,0)|^{2}+1\right),
$$

where $C$ is a universal constant.

\section{References}

[1] V. Bally, Approximation scheme for solutions of BSDE, Backward stochastic differential equations (N. El Karoui and L. Mazliak, eds.), Pitman Res. Notes Math. Ser., vol. 364, Longman, Harlow, 1997, pp. 177-191. 
[2] D. Chevance, Numerical methods for backward stochastic differential equations, Numerical methods in finance, Cambridge Univ. Press, Cambridge, 1997, pp. 232-244.

[3] D. Chevance, Résolution numériques des équations différentielles stochastiques rétrogrades, Ph.D. thesis, Université de Provence - Aix-Marseille I, Marseille, 1997.

[4] F. Coquet, V. Mackevičius, and J. Mémin, Corrigendum to: "Stability in $\mathbf{D}$ of martingales and backward equations under discretization of filtration" [Stochastic Processes Appl. 75 (1998)], Stochastic Process. Appl. 82 (1999), no. 2, 335-338.

[5] F. Coquet, J. Mémin, and L. Słomiński, On weak convergence of filtrations, Séminaire de Probabilités, XXXV, Lecture Notes in Math., Springer, Berlin, to appear.

[6] J. Douglas, J. Ma, and Ph. Protter, Numerical methods for forward-backward stochastic differential equations, Ann. Appl. Probab. 6 (1996), no. 3, 940-968.

[7] N. El Karoui, S. Peng, and M.-C. Quenez, Backward stochastic differential equations in finance, Math. Finance 7 (1997), no. 1, 1-71.

[8] J. Jacod, Convergence en loi de semimartingales et variation quadratique, Séminaire de Probabilités, XV, Lecture Notes in Math., vol. 850, Springer, Berlin, 1981, pp. 547-560.

[9] J. Ma, Ph. Protter, and J. Yong, Solving forward-backward stochastic differential equations explicitly - a four step scheme, Probab. Theory Related Fields 98 (1994), no. 3, 339-359.

[10] J. Ma and J. Yong, Forward-backward stochastic differential equations and their applications, Lecture Notes in Math., vol. 1702, Springer-Verlag, Berlin, 1999.

[11] J. Mémin and L. Słomiński, Condition UT et stabilité en loi des solutions d'équations différentielles stochastiques, Séminaire de Probabilités, XXV, Lecture Notes in Math., vol. 1485, Springer, Berlin, 1991, pp. 162-177.

[12] E. Pardoux and S. Peng, Adapted solution of a backward stochastic differential equation, Systems Control Lett. 14 (1990), no. 1, 55-61.

[13] E. Pardoux and S. Peng, Backward stochastic differential equations and quasilinear parabolic partial differential equations, Stochastic partial differential equations and their applications (Charlotte, NC, 1991) (B. L. Rozovskii and R. B. Sowers, eds.), Lecture Notes in Control and Inform. Sci., vol. 176, Springer, Berlin, 1992, pp. 200-217.

[14] S. Peng, Probabilistic interpretation for systems of quasilinear parabolic partial differential equations, Stochastics Stochastics Rep. 37 (1991), no. 1-2, 61-74.

[15] L. Słomiński, Stability of stochastic differential equations driven by general semimartingales, Dissertationes Math. (Rozprawy Mat.), vol. 349, Polish Academy of sciences, Warszawa, Poland, 1996. 